\title{
En niños, la BCG también disminuiría el riesgo de infección por tuberculosis
}

Soysal A et al. Lancet 2005;366:1443-1451.

Objetivo

Investigar factores de riesgo asociados a la infección por M. Tuberculosis en niños convivientes con pacientes bacilíferos positivos y el efecto de la vacunación con BCG.

\section{Diseño}

Estudio prospectivo basado en la comunidad

\section{Lugar}

Estambul, Turquía.

\section{Pacientes}

979 niños de 3 a 16 años contactos convivientes de 414 adultos con tuberculosis (TBC) con esputo positivo.

\section{Evaluación de factores pronósticos}

Edad, sexo, número de contactos, vacuna BCG, caso índice con TBC multiresistente, parentesco, exposición y factores socioeconómicos (ingreso, empleo, educación de ambos padres) y tabaquismo en la casa.

\section{Medición de resultados principales}

El estatus de infección fue evaluado con reacción de Mantoux (PPD) tradicional y con ELISpot, test basado en la ídentificación de linfocitos $T$ con respuesta específica a antígenos del $M$. Tuberculosis que no se altera con la BCG.

\section{Resultados principales}

En análisis multivariable*, sólo la edad (a mayor edad mayor exposición a TBC fuera de la casa), la intensidad de exposición y la ausencia de cicatriz de BCG fueron identificados como factores independientes* del riesgo de infección asociado a la exposición.

Tabla. Riesgo de infección por TBC en niños vacunados con BCG

\begin{tabular}{|c|c|c|c|}
\hline Variable & Odids Ratio & IE95\% & p \\
\hline Cicatriz de vacuna BCG & 0,60 & 0,43 a 0,83 & $<0,003$ \\
\hline
\end{tabular}

\section{Conclusiones}

En los niños, la BCG no solo reduce el desarrollo de formas diseminadas de tuberculosis sino también disminuye el riesgo de infección posterior a una exposición con un caso bacilífero positivo.

\section{Comentario}

La tuberculosis sigue siendo la enfermedad infecciosa que causa mayor morbi-mortalidad en el mundo. La incidencia estimada es de aproximadamente ocho millones de casos nuevos por año y casi dos millones de muertes. Más del $90 \%$ de estos casos ocurren en los países subdesarrollados. ${ }^{1,2}$ Alineados con las recomendaciones de la Organización Mundial de la Salud, los programas de control de la TBC se basan en el diagnóstico precoz y tratamiento efectivo de los enfermos y en la prevención del desarrollo de TBC activa entre los infectados. ${ }^{3}$ La BCG ha sido y sigue siendo una de las estrategias de control más utilizadas en el mundo y es parte de la recomendación de vacunación obligatoria dentro de los primeros tres meses de vida en los países de alta y mediana incidencia y prevalencia de TBC. Un reciente análisis de costo-efectividad describe a la BCG como una de las estrategias más costo-efectivas en la prevención de la TBC miliar y meningitis tuberculosa en niños. ${ }^{1.4} \mathrm{Al}$ estar expuesto a un caso fuente, la precisa identificación de los contactos infectados reviste una importancia mayor, ya que el tratamiento profiláctico con isoniazida reduce sustancialmente el riesgo de desarrollo ulterior de enfermedad activa y esto es especialmente cierto en los niños. El tiempo de contacto con un caso fuente estimado para adquirir la infección es de aproximadamente cinco días. ${ }^{5} \mathrm{El}$ presente estudio fue realizado en Estambul, que presenta una incidencia anual de TBC de 40/100.000 y vacunación universal con BCG en los niños recién nacidos, características muy comparables a las de la Argentina. El uso de ELISpot, más sensible y específico que la centenaria PPD para el diagnóstico de infección, permite identificar en forma más confiable a los niños infectados con $M$ Tuberculosis, ya que no presenta reacción cruzada con la $B C G^{6}$. Esta particularidad permitió ver que los niños vacunados con BCG tuvieron significativamente menos riesgo de infección (ELISpot positivo) que los no vacunados, ajustando por otros factores claramente vinculados como intensidad de la exposición y edad. En los países desarrollados, el uso de ELISpot puede implicar un paso adelante en el control de la TBC latente, ya que permite concentrar las estrategias profilácticas más específicamente sobre la población infectada. En nuestros países, en los que el uso de BCG está difundido, el efecto sobre la reducción del riesgo de infección en niños expuestos resulta muy interesante, ya que hasta hoy sólo se reconocía el efecto sobre el desarrollo de formas diseminadas graves $\sin$ mostrar efectividad en los adultos. Por otro lado, la mayor sensibilidad* del ELISpot ayudará a disminuir los falsos negativos de la PPD, especialmente en la población VIH positivo e inmunodeprimidos, quienes son los que conllevan más riesgo de desarrollar enfermedad activa ${ }^{3}$. Si bien el ELISpot se ha podido utilizar con éxito en regiones de bajos recursos, todavía se encuentra en etapa de aprobación para su uso masivo, y no contamos aún con información de costos de su implementación.

Por último, el conocimiento sobre la respuesta de linfocitos $T$ específicamente dirigidos a proteínas del M. Tuberculosis abre enormes perspectivas sobre el desarrollo de vacunas que las utilizan como antígenos específicos. Algunos ensayos clínicos en curso se muestran muy alentadores en la generación de la respuesta inmune alcanzada con esta nueva vacuna utilizada en pacientes ya vacunados con BCG.

\section{Conclusiones del comentador}

Si bien en nuestros países la estrategia prioritaria sigue siendo la identificación y tratamiento supervisado efectivo de los casos activos, este efecto no reportado previamente de la BCG reafirma aún más su indicación masiva; y junto con el conocimiento sobre las nuevas vacunas aparecen como promisorias en el combate contra la TBC.

\section{Fernando Rubinstein [ Unidad de Medicina familiar y Preventiva. Hospital Italiano de Buenos Aires. ]}

Rubinstein F. En niños, la BCG también disminuiría el riesgo de infección por tuberculosis. Evid. act. pract. ambul. 9(2):38 Mar-Abr.2006. Comentado de: Soysal,A, Millington, K, Bakir,M. Effect of BCG vaccination on risk of mycobacterium tuberculosis infection in children with household tuberculosis contact: a prospective community-based study. Lancet 2005;366:1443-1451. PMID: 16243089.

\section{Referencias}

1. F Rubinstein et al. Tuberculosis. Medicina Familiar y Práctica Ambulatoria. 2a edición Editorial Panamericana 2006 Pág. $921-938$

2. TB or not TB? A new test for the disease of the pharaons.2003 http//www.wellcome.ac.uk/doc_WTD004528.htm

3. A. Lalvani. Spotting latent infection: the path to better tuberculosis control. Thorax 2003;58:916-918

4. B Bourdin Trunz, PEM Fine, C Dye. Effect of BCG vaccination on childhood tuberculous meningitis and miliary tuberculosis worldwide: a metaanalysis and assessment of cost-eff ectiveness Lancet 2006; 367: 1173-80

5. 130 hours: exposure time needed to contract tuberculosis. $2003 \mathrm{http} / \mathrm{www}$. wellcome.ac.uk/doc_WTD002828.htm

6. Shams H, Weis S Klucar P, et al. Enzyme Linked Immunospot and tuberculin skin testing to detect Latent Tuberculosis Infection. Am J Respir Crit Care Med. 172; 1161-1168, 2005

7. Tuberculosis vaccine trials success. 2004. www.wellcome.ac.uk/doc_WTX022716.html 